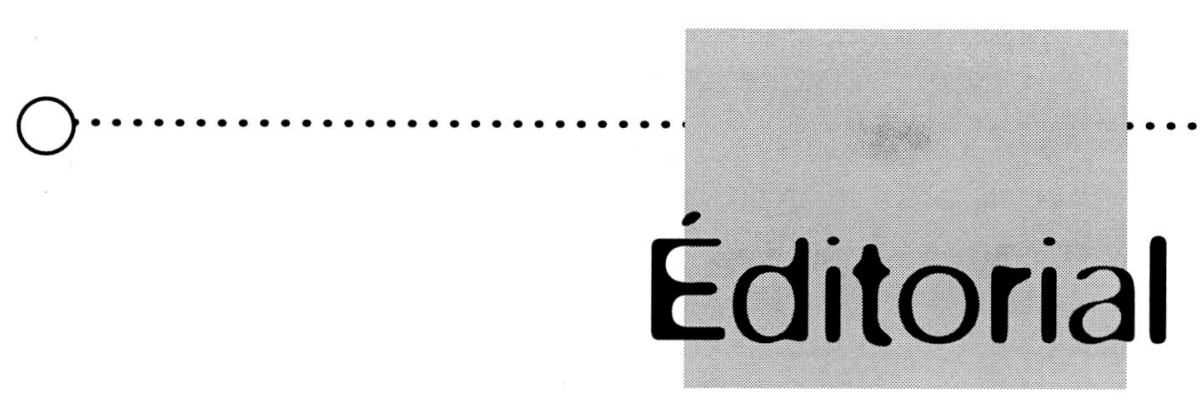

\title{
Administrer la psychiatrie ?
}

\author{
Bernard ODIER*
}

* Psychiatre

des Hôpitaux,

Médecin-chef de secteur, ASM13, 11 , rue Albert Bayet, 75013 Paris, France.

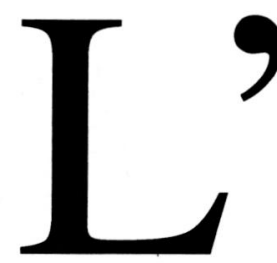

évolution préoccupante actuelle de la psychiatrie, et en particulier de la psychiatrie publique, conduit à s'interroger sur l'existence d'une politique dans ce domaine. Les mutations socio-économiques à l'œuvre dans le champ de la psychiatrie depuis environ vingt ans sont si considérables que l'on pourrait s'attendre à qu'elles aient été élaborées et planifiées dans un vaste plan de restructuration. Celui-ci n'existe pas ; il n'y a pas eu de grand horloger pour programmer ce déclin.

D'ailleurs, si les structures de soin se défendent comme des beaux diables pour résister au cercle vicieux qui voit s'enchaîner manque de personnel, régression des pratiques, et perte d'attractivité pour les soignants, le secteur administratif et chargé de la gestion de l'appareil de soin ne connaît pas la même tendance. Une véritable technostructure se développe lentement depuis une quinzaine d'années, qui associe des formes modernisées d'inspection des établissements (à l'IGAS ${ }^{1}$ s'est ajoutée la Mission Appui, puis l'ANAES ${ }^{2}$ avec l'accréditation), d'analyse de gestion (quelle $\mathrm{ARH}^{3}$ ne dispose pas de sa cellule statistique établissant à longueur d'années des supports chiffrés pour toutes sortes de comparaisons ?), et d'analyse des coûts (la comptabilité analytique étant mobilisée aujourd'hui pour le $\mathrm{PMSI}^{4}$, et demain pour la tarification à l'activité). Un véritable paradoxe se met en place, qui voit se conjuguer un déclin subi et le développement des éléments d'une maîtrise « sur le papier »; l'ensemble appelant d'une

1. IGAS : Inspection Générale des Affaires Sociales.

2. ANAES : Agence Nationale de l'Accréditation et de l'Évaluation Sanitaire.

3. ARH : Agence Nationale de l'Hospitalisation.

4. PMSI : Programme Médical Standardisé Informatisé. seule voix au développement d'une médecine contemplative, avec plus de veille sanitaire, plus de surveillance des produits de santé, plus d'épidémiologie, etc.

Ce qu'il faut bien appeler un processus de bureaucratisation se déploie, sensible par exemple dans l'augmentation continue de la longueur des textes législatifs et réglementaires concernant la psychiatrie. Comparer la brièveté de la Circulaire de 1960 (à l'origine du développement de la psychiatrie de secteur) et la longueur des attendus de l'arrêté du 14 mars 1986 (décrivant dans le détail les différentes "structures du secteur ») permet de pointer un des mécanismes du tarissement de l'élan fondateur. Progressivement, les chefs de service se sont vus dicter dans le détail les modalités d'engagement de leurs moyens. Un lent et silencieux glissement des responsabilités les a progressivement dépossédés de leur pouvoir, celui d'agencer de façon ajustée les réponses soignantes aux caractéristiques locales de la population et du territoire dont ils devaient organiser la desserte. Dans le meilleur des cas, l'exercice de cette responsabilité faisait du médecin-chef de secteur un acteur de la vie politique locale reconnu, et ses avis étaient recherchés.

Un découplage avec la vie politique locale s'est produit quand, au nom d'une généralisation (la « légalisation » du secteur en 1985), les budgets dits extra-hospitaliers ont cessé d'être votés par les Conseils Généraux. Progressivement, une dévitalisation des relations avec les élus s'est produite, qui s'est encore accentuée lorsque la tutelle des établissements a été confiée en 1995 aux A.R.H., achevant ainsi une séparation entre le niveau des responsabilités politiques départementales et l'évolution des structures de soin. Le dernier signe, bien menacé, de cette solidarité entre représentation démocratique et structures de soin tient dans la présence d'élus au sein des 
Conseils d'administration. Les gestionnaires d'inspiration ultra-libérale la qualifient d'archaïsme.

L'épisode de surmortalité lié à la canicule pendant l'été dernier n'a peut-être pas été suffisamment commenté sous l'angle de ce qu'il révélerait d'une coupure grandissante entre l'évolution des structures de soin et celle des besoins de la population. Le réseau ville-hôpital spécialisé que constituait avant l'heure la psychiatrie de secteur est bâti pour favoriser l'ajustement de la réponse. Encore faut-il que les secteurs aient gardé suffisamment de marge de manœuvre et d'autonomie.

Sous ce rapport, l'évolution actuelle de la doctrine administrative est hésitante. D'un côté, on brandit des chiffres soulignant les disparités de moyens dont disposent les secteurs pour étayer l'idée d'un échec de la psychiatrie de secteur; de l'autre, dans la mouvance de la mise en place d'une Europe des marchés, on prône la décentralisation qui généralisera l'inégalité dans l'accès aux soins. L'idée d'équité n'a pas d'autre fonction idéologique que de se substituer insidieusement, pour l'affaiblir, à l'idéal d'égalité de tous dans l'accès aux soins (et beaucoup d'entre nous, dans leur travail quotidien, donnent corps à cette utopie) ; l'idée de subsidiarité propose quant à elle de rendre en quelque sorte facultative pour les états une politique de santé.

Les psychiatres savent qu'une partie de leurs patients ne mobilise pas spontanément autour d'eux tolérance et solidarité. Pour les plus malades d'entre eux, un certain nombre de caractéristiques cliniques, de la destructivité au clivage en passant par l'identification projective, mettent à mal leur entourage et leur contexte social. La solidarité humaine qu'exige leur état est une solidarité non désirée. Avons-nous enregistré trop rapidement comme un progrès l'abandon des termes un peu martiaux de "lutte contre les fléaux sociaux » à l'enseigne desquels l'état organisait la « lutte contre les maladies mentales »? Si cette terminologie peut sembler aujourd'hui décalée pour notre Société si pressée de se montrer compréhensive, elle avait l'avantage de situer du côté de l'État la garantie que ces situations humaines ne donneraient pas lieu à la cascade de désinvestissements, de démissions, de petites lâchetés qui se multiplient aujourd'hui, et dont une traduction criante nous est renvoyée par la multiplication des psychotiques vivant à la rue et par le retour des régicides.

Pierre Bourdieu nous enseignait que la révolution conservatrice (et pas la prétendue Réforme !) à l'œuvre actuellement voit l'État se dessaisir de ce qu'il tient dans sa main gauche (le social, l'économique), pour se centrer sur sa main droite (c'est-àdire ses fonctions régaliennes : l'ordre, la sécurité, la justice). Mais, précise-t-il, il doit rattraper de sa main droite une partie de ce qu'il lâche de la main gauche. Ainsi dans le domaine de la Santé, et de la santé mentale en particulier, nous observons une évolution en peau de chagrin de la desserte interdisant progressivement toute action de Prévention, tandis que de rares « crédits fléchés » sont promis à des « actions ciblées » en faveur des coupables, voire des victimes. Cherchez l'erreur.

$4^{\mathrm{e}}$ Colloque pluridisciplinaire

\section{Actualités sur les explorations du cerveau et du psychisme de l'enfant}

Journée d'étude organisée par Julien Cohen-Solal, Philippe Evrard et Bernard Golse

Samedi 19 juin 2004

Le Sénat - Palais du Luxembourg - Salle Clémenceau

\section{Renseignements}

Christelle Mourinet et Françoise Richard

Secrétariat de pédopsychiatrie, Hôpital Necker Enfants-Malades,

149, rue de Sèvres, 75743 Paris Cedex 15, France.

Tél. : 01444959 18/19. 